\title{
In-Vivo Estimates of Axonal Characteristics Using Optimized Diffusion MRI Protocols for Single Fibre Orientation
}

\author{
Torben Schneider ${ }^{1}$, Claudia A.M. Wheeler-Kingshott ${ }^{1}$, \\ and Daniel C. Alexander ${ }^{2}$ \\ 1 NMR Unit, Department of Neuroinflammation, UCL Institute of Neurology, \\ University College London, UK \\ T.Schneider@ion.ucl.ac.uk \\ 2 Centre for Medical Image Computing, Department of Computer Science, University \\ College London, UK
}

\begin{abstract}
This work presents diffusion MR protocols that allow estimation of axonal parameters like diameter and density in the live human brain. Previous approaches demand very high field experimental systems or suffer from long acquisition times and are therefore impractical for use in clinical studies. We propose a method that significantly reduces scan time by making use of the a-priori known fibre orientation in structures with well defined single fibre (SF) organisation like the corpus callosum (CC) and produces protocols that can be performed in under 25 minutes on a standard clinical system. Results from a computer simulation experiment show that our SF protocols can generate parameter estimates with similar precision to previously proposed orientation invariant (OI) protocols. Furthermore, we acquire the 20 minute long SF protocol and the 1 hour long OI protocol in a scan/rescan study on two healthy subjects and compare the axonal parameter maps from both protocols.
\end{abstract}

\section{Introduction}

Diffusion weighted imaging (DWI) is an MRI technique that is sensitive to the random motion of water molecules and thus provides an insight into the microstructural properties of biological tissue. In recent years, numerous studies have demonstrated the great potential of DWI measures for the investigation of pathological and developmental changes of the human central nervous system. One of the most commonly used DWI method is diffusion tensor imaging (DTI) [1]. DTI allows the computation of useful indices, e.g., mean diffusivity or anisotropy of diffusion in each voxel but those measures are unspecific to individual microstructural characteristics.

Recently, Assaf et al[2] developed the AxCalibre model of cylindrical axons with gamma distributed radii to estimate axon diameter distributions in white matter tissue. The method is validated in in-vitro optic and sciatic nerve samples and estimated parameters show good correlation with corresponding histology. Barazany et al[3] applies the AxCalibre approach to image axon size distributions

T. Jiang et al. (Eds.): MICCAI 2010, Part I, LNCS 6361, pp. 623-630 2010

(C) Springer-Verlag Berlin Heidelberg 2010 
in the corpus callosum of live rat brain. However, scan times are long and the high $7 \mathrm{~T}$ magnetic field and maximum gradient strength $(400 \mathrm{mT} / \mathrm{m})$ are impossible to achieve on a live human scanners, that typically operate at $1.5-3 \mathrm{~T}$ and can achieve maximum gradient strength between $30-60 \mathrm{mT} / \mathrm{m}$. Alexander et al 4 ] demonstrates measurements of axon diameter and density in the live human brain on a standard clinical scanner using a simplified AxCalibre model and multi shell high angular resolution diffusion imaging (HARDI). The method relies on an experiment design optimization [5] to achieve sensitivity without the need of high gradient strengths and long acquisition times. The use of HARDI permits orientation invariant (OI) estimation of microstructure parameters over large areas of the brain with a range of different fibre orientations. However, with scan time close to an hour this approach is still impractical for clinical studies. Furthermore, with increasing scan time subjects are more likely to move during the acquisition, which results in a loss of signal quality and decreases the accuracy of parameter estimation.

Our aim in this study is to reduce total scan time to a clinical feasible maximum of 20-25 minutes while maintaining accuracy of the parameter estimation. To attain the reduction we discard the requirement for orientational invariance and focus on structures with known fibre orientation such as the corpus callosum similar to earlier studies [23]. We modify the existing protocol optimization framework [5] to incorporate this a-priori information about fibre organisation and define single fibre (SF) protocols that measure only the diffusion signal perpendicular and parallel to the known fibre orientation. We use computer simulations to compare the SF and the OI approach and perform a scan/rescan experiment on two healthy volunteers to investigate feasibility of estimating microstructural parameters in-vivo.

\section{Methods}

\subsection{Tissue Model}

As in Alexander et al 4, we model diffusion in a white matter voxel as a combination of water particles trapped inside three different compartments:

1. Intra-axonal water experiencing diffusion restricted inside cylindrical axons with equal radius $R$ as in 8

2. Extra-axonal water that is hindered due to the presence of adjacent axons. Diffusion is approximated by a diffusion tensor, with parallel diffusion coefficent $d_{\|}$in the direction of the cylinders and symmetric diffusion $d_{\perp}$ in the perpendicular directions.

3. Water that experiences unhindered diffusion, e.g., in the cerebro-spinal fluid (CSF), modeled by an isotropic Gaussian distribution of displacements with diffusion coefficient $d_{I}$.

To reduce the number of free parameters in the model, we express $d_{\perp}$ by using the tortuosity model of Szafer et al 9 . 
The diffusion weighted MR signal is measured by the pulsed gradient spin echo (PGSE) method, which has has the following free parameters: diffusion sensitising gradient vector $\boldsymbol{G}$ of strength $|\boldsymbol{G}|$ and duration $\delta$, and diffusion time $\Delta$. We combine the analytic expressions for the PGSE signal of each compartment $S_{i}(\boldsymbol{G}, \delta, \Delta)$ with $i$ in $1, \cdots, m$ to approximate the total signal $S$ in each voxel by:

$$
S(\boldsymbol{G}, \delta, \Delta)=S_{0} \sum_{i=1}^{3} f_{i} S_{i}(\boldsymbol{G}, \delta, \Delta),
$$

where $S_{0}$ is the MR signal with no diffusion weighting and $f_{i}$ is the volume fraction of each tissue compartment with $0 \leq f_{i} \leq 1$ and $\sum_{i=1}^{3} f_{i}=1$.

\subsection{Protocol Optimisation}

The aim of this work is to use a model-based imaging approach in each voxel to fit a set of MR signals acquired with different combinations of PGSE parameters to the tissue model parameters. A minimum of 6 different diffusion MR signals are required to estimate all model parameters but usually we acquire more signals to overdetermine the solution and add noise control. We define a set of PGSE parameters that acquires $N$ different MR signals as protocol

$$
\mathcal{P}=\left\{\boldsymbol{G}_{1}, \delta_{1}, \Delta_{1} \cdots, \boldsymbol{G}_{N}, \delta_{N}, \Delta_{N}\right\}
$$

As mentioned above, the aim of the optimisation algorithm [5] is to find the protocol $\mathcal{P}$, that allows the most accurate estimation of the tissue model parameters under given hardware and time constraints. The Fisher information matrix (FIM) provides a lower bound on the inverse covariance matrix of parameter estimates, i.e., the $\mathcal{P}$ that maximizes the FIM will maximize the precision of those estimates. We use the d-optimality criterion [6], which is defined as the determinant of the inverse FIM of protocol $\mathcal{P}$ and tissue model parameters $\boldsymbol{\theta}$ :

$$
D(\boldsymbol{\theta}, \mathcal{P})=\operatorname{det}\left[\left(\boldsymbol{J}^{T} \boldsymbol{\Omega} \boldsymbol{J}\right)^{-1}\right],
$$

where $\boldsymbol{J}$ is the $N \times 6$ Jacobian matrix with the $i j$ st element $\partial S\left(\boldsymbol{G}_{i}, \delta_{i}, \Delta_{i}\right) / \partial \boldsymbol{\theta}_{j}$. In the original approach $\boldsymbol{\Omega}=\operatorname{diag}\{1, \cdots, 1\}$. Following [5], we use a stochastic optimization algorithm [7] that returns $\mathcal{P}^{\prime}$ with minimal $D$ among all possible $\mathcal{P}$ with respect to the given scanner hardware limits.

In the original approach, $N$ is chosen to account for the acquisition time limit. The acquisition is divided in $M$ sets of different PGSE settings with gradient directions in each set being fixed. However, in this approach a decrease of the total number of acquisitions $N$ must reduce the angular resolution of gradient direction sampling, which will increase the uncertainty in fibre direction estimates and thus tissue parameter measures. The OI approach also requires that $N$ and $M$ are known so that for every combination of $N$ and $M$ the full optimization algorithm has to be performed. Furthermore this method does not reward protocols that sample more important measurements more heavily. 
In this work we introduce the asymptotic SF protocol optimisation. We focus on specific structures with known fibre orientation like the CC. This avoids the need for high angular resolution, which potentially dramatically reduces the number of required measurements. As in [23, we constrain most measurements in the protocol to have gradient direction perpendicular to the fibre bundles, but we include one measurement in the parallel direction for the estimation of diffusivity along the axons. We extend the algorithm to optimize $M$ different PGSE settings and include $\boldsymbol{\Omega}=\operatorname{diag}\left\{w_{1}, \cdots, w_{M}\right\}$ with $\sum_{m=1}^{M} w_{m}=1$ in the optimisation of Eq. 3. The weighting factors $w_{m}$ reflect how important each measurement is, i.e. how often it should be sampled relative to the other measurements. For any given $N$ we can calculate the number of measurements for each element of $\mathcal{P}$ by $N_{m}=w_{m} N$, hence the resulting protocols are independent of $N$.

\subsection{Model Fitting}

We use the three stage fitting algorithm as described by Alexander et al [4, to fit the tissue model to the acquired MR signal in each voxel. We increase stability by fixing $d_{\|}$to $1.7 \cdot 10^{-9} \mathrm{~m}^{2} \mathrm{~s}^{-1}$ and $d_{I}$ is fixed to $3.0 \cdot 10^{-9} \mathrm{~m}^{2} \mathrm{~s}^{-1}[234$. The objective function is defined as the maximum likelihood of model parameters given the observed MR signals under Rician noise $(\sigma=0.05)$. An initial estimation is found using a coarse grid search algorithm over a set of physiologically possible parameters. Then a gradient descent algorithm further refines the parameter estimates. Finally a Markov Chain Monte Carlo (MCMC) algorithm with a burn-in of 2000, 50 samples at an interval of 200 provides posterior distributions of the parameters $f_{1}, f_{2}$ and the axon radius $r$. An average over the MCMC samples provides the final parameter estimates. We report the axon diameter index $a=2 r$ and the axon density index $\rho=4 f_{1} \pi^{-1} r^{-2}$.

\section{$3 \quad$ Experiments and Results}

We motivated our protocol optimization by the aim to produce protocols that can be performed on a typical human scanner in a clinically feasible time. To validate our approach, we generate optimized protocols for a clinical 3T Philips Achieva scanner with a maximum gradient strength of $\left|\boldsymbol{G}_{\max }\right|=60 \mathrm{mT} / \mathrm{m}$. We use the asymptotic optimization to generate $\mathrm{SF}$ protocols that can be performed in 20 minutes with a total number of acquisitions $N=90\left(\mathrm{SF}_{90}\right)$. For comparison with previous studies, we also generate an OI protocol using $N=360\left(\mathrm{OI}_{360}\right)$ and an $\mathrm{SF}$ protocol with the same number of acquisitions $\left(\mathrm{SF}_{360}\right)$. The resulting protocols are presented in table 1 . For the $\mathrm{SF}_{90}$ and $\mathrm{SF}_{360}$ protocols we set $M=8$ but only sequences with $w>0$ are reported. The $\mathrm{OI}_{360}$ protocol optimisation uses $M=4$ and report the three unique PGSE parameter settings.

\subsection{Simulations}

We use the free diffusion simulation of Hall and Alexander [10], which performs a Monte Carlo (MC) simulation of water particles in packed cylinders. We use 
Table 1. PGSE settings of $\mathrm{SF}_{90}, \mathrm{SF}_{360}$ and $\mathrm{OI}_{360}$ protocols. $\perp$ and $\|$ mark acquisitions perpendicular and parallel to the fibre bundles.

(a) $S F_{360}$ and $S F_{90}$ protocols

\begin{tabular}{lllllll} 
& \multicolumn{1}{c}{$\Delta$} & $\delta$ & $G$ & $b$ \\
$N_{m}$ & {$[m s]$} & {$[m s]$} & {$[m T / m]\left[s / m^{2}\right]$} \\
\hline 70 & 18 & 0 & 0 & 0 & 0 & \\
72 & 17 & 33.0 & 14.5 & 36.8 & 550 & $\|$ \\
38 & 10 & 22.4 & 15.9 & 60.0 & 1114 & $\perp$ \\
45 & 11 & 29.3 & 22.8 & 60.0 & 2908 & $\perp$ \\
68 & 17 & 48.0 & 26.6 & 43.7 & 3666 & $\perp$ \\
67 & 17 & 40.5 & 34.0 & 60.0 & 8692 & $\perp$ \\
\hline 360 & 90 & & & &
\end{tabular}

(b) $\mathrm{OI}_{360}$ protocol

\begin{tabular}{lllll}
\multicolumn{1}{c}{$\begin{array}{lll}\Delta \\
N_{m}\end{array}$} & $\delta$ & $G$ & $b$ \\
\hline 71 & 0 & 0 & 0 & 0 \\
101 & 19.2 & 11.7 & 60.0 & 540 \\
107 & 38.2 & 12.5 & 47.8 & 870 \\
81 & 29.1 & 21.6 & 60.0 & 2634 \\
\hline 360 & & &
\end{tabular}

the 44 synthetic white matter substrates from Alexander et al [4] with diameter distributions and packing densities similar to previously reported histology studies. We perform the MC simulation with 50000 walkers and 20000 time steps for each protocol. For each substrate we generate 10 sets of noise-free MR signals and add Rician noise of $\sigma=0.05$, resulting in total of 440 sets of noisy MR signals. For each protocol we apply the model fitting procedure to the 440 sets of MR signals and retrieve the tissue model parameters.

To compare the axon distributions with the estimated axon diameter index $a$ we have to take into consideration that the contribution of each axon to the MR signal depends its volume and is proportional to the square of its diameter. As in [4 we correlate the estimated axon diameter index $a$ with the weighted axon diameter average $\hat{a}=\hat{f} / \int p(\alpha) \alpha^{3} \mathrm{~d} \alpha$, where $p$ is the true distribution of axon diameter $\alpha$ and $\hat{f}$ is the intracellular volume fraction $\hat{f}=\int p(\alpha) \alpha^{2} \mathrm{~d} \alpha$.

\subsection{MRI Experiment}

The $\mathrm{SF}_{90}$ and $\mathrm{OI}_{360}$ protocols (see table 1) are implemented on a $3 \mathrm{~T}$ Philips Achieva scanner to test the clinical viability of the 20 minute $\mathrm{SF}_{90}$ protocol and compare it to the three times longer $\mathrm{OI}_{360}$ protocol. Diffusion weighted MR images of two healthy volunteers (male 32yo, female 25yo) are acquired using a cardiac-gated EPI sequence with the following imaging parameters: 10 slices, slice thickness $=5 \mathrm{~mm}$, in-plane resolution $=128 \times 128\left(\mathrm{FOV}=35 \mathrm{x} 35 \mathrm{~mm}^{2}\right)$, $\mathrm{TR}=7 \mathrm{RR}, \mathrm{TE}=125 \mathrm{~ms} / \mathrm{TE}=100 \mathrm{~ms}$ for $\mathrm{SF}_{90}$ and $\mathrm{OI}_{360}$ respectively. We position the centre slice so that it is aligned with the mid-sagittal body of the CC to be able to acquire DWI measurements perpendicular and parallel to the fibres of the CC. $\mathrm{SF}_{90}$ acquisition is repeated twice on two separate days for each subject to investigate the reproducibility of the estimated parameter maps.

\subsection{Results}

Figure 1 presents the results from fitting the model to the synthetic MC data sets as described above. For all three protocols we plot the fitted axon diame- 

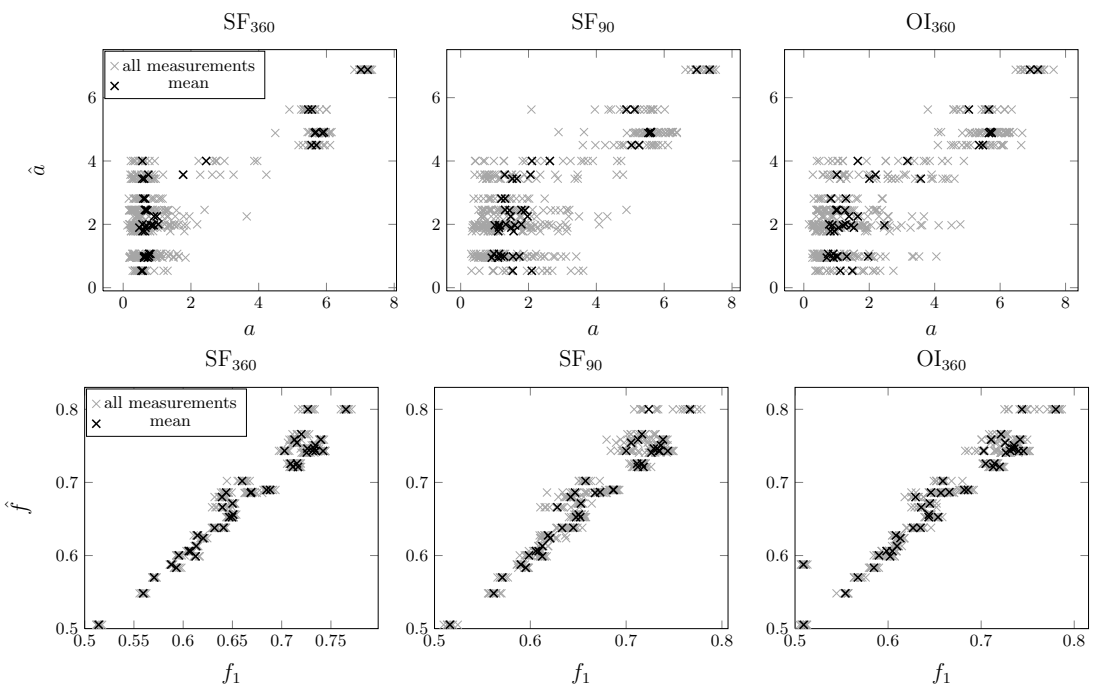

Fig. 1. Scatter plots of estimated tissue model parameters $a$ and $f_{1}$ (grey) and and mean $a$ and $f_{1}$ over 10 replications (black) against true $\hat{a}$ and $\hat{f}$ of the MC substrates

ter index $a$ against $\hat{a}$ and the intra-cellular volume fraction $f_{1}$ against the true intra-cellular volume fraction $\hat{f}$ for all 440 noisy sets of MR signals. We also compute the mean over the 10 replications for each of the 44 unique substrates and display them in the same plot. The bottom row of Fig 1 shows that all protocols estimated the volume fraction accurately with little variance. Further, all protocols estimate larger $a$ that agree with $\hat{a}$. The estimated $a$ varies arbitrarily between $0-2 \mu m$ for $\hat{a} \lesssim 3 \mu m$. Thus smaller $\hat{a}$ can be distinguished from larger ones but not accurately measured. This is because the limited maximal gradient strength that does not attenuate the signal from water inside axons of diameter $<2 \mu \mathrm{m}$. Despite the limitation, the trends of $a$ agree with the true values for $\hat{a}$ and suggest that the index $a$ is a useful discriminator of axon diameter distributions. $\mathrm{SF}_{360}$ estimates both indices more accurately than $\mathrm{OI}_{360}$ and variations among the 10 estimates in each substrate are smaller. $\mathrm{SF}_{90}$ and $\mathrm{OI}_{360}$ appear to have similiar accuracy and precision in estimating $\hat{a}$ and $\hat{f}$. This suggests that we can reduceby a third by exploiting a-priori known fibre orientation while maintaining similar quality of parameter estimates.

Figure 2 shows maps of $a$ and $\rho$ in the centre slice of the CC for all acquisitions in two volunteers. From previous histological studies [11 we expected low axon diameter and high density in the splenium and genu and higher axon diameters with lower density in the body of the CC. As predicted by the MC simulations (see also [4), all protocols overestimated $a$ because of the lack of sensitivity to lower diameters. The high-low-high trend in $a$ and low-high-low trend of $\rho$ can be observed in both subjects in $\mathrm{OI}_{360}$ results but are less apparent in $\mathrm{SF}_{90}$ scans. The worst case is is $\mathrm{SF}_{90}$ of subject 1, which presents very noisy parameter maps. This is likely to be caused by a misalignment with the true 


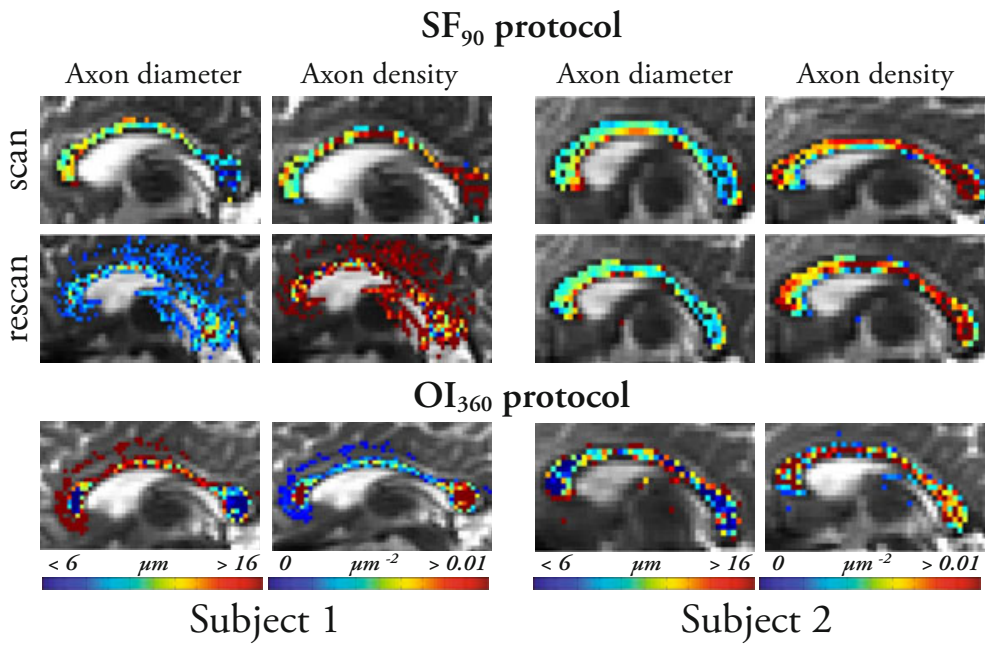

Fig. 2. Color coded parameter maps of $a$ and $\rho$ in the centre slice of the CC in two subjects. Scan and rescan results for the $\mathrm{SF}_{90}$ are shown together with results from the $\mathrm{OI}_{360}$ acquisition.

fibre direction of the $\mathrm{CC}$ and the gradient directions, which demonstrates the sensitivity of the SF protocol to accurate positioning. Furthermore, all SF scans consistently produce larger estimates of $a$ than $\mathrm{OI}_{360}$. Variation in true fibre orientation is again the likely explanation. Unlike the SF protocols, the OI protocol can better compensate for this variation because of the high angular gradient sampling. However, despite the limitations, the results of subject 2 demonstrate reproducible estimates of $a$ and $\rho$. This suggests that with accurate positioning, the 20 minute $\mathrm{SF}_{90}$ protocol is able to produce comparable parameter maps to $\mathrm{OI}_{360}$, which requires more than three times the scan time.

\section{Conclusion}

In this work we propose optimized diffusion MRI protocols that use the known fibre orientation in specific structures like the $\mathrm{CC}$ and allow us to estimate indices of axon diameter and density in the live human brain. We develop a new optimization algorithm that overcomes several limitations of previous approaches and produces DWI protocols that can be acquired in under 20 minutes. While previous protocols were too time consuming for clinical practise, the short acquisition time of our protocols opens the possibility to be included in a variety of studies. Experiments on synthetic data show that our protocols can provide axon diameter and density indices with similar variance to those from longer orientational invariant protocols. In-vivo scans on two healthy volunteers show the potential of our method to produce parameter maps of axon diameter and density that agree with the general histologic trend but also reveal the limitations caused by misalignment and variation in fibre orientation compared to the 
longer OI protocol. If such protocols are to be used, great care must be taken to align gradient directions with the fibre orientation. Future work aims to account for uncertain or erroneous fibre orientation by incorporating some tolerance for fibre orientation variation in the optimisation.

Acknowledgements. Thanks to Dr Hubbard for support in implementing the protocols. TS is sponsored by the ISRT. The NMR Unit at the UCL Institute of Neurology is supported by the MS society of Great Britain and Northern Ireland. DCA is funded by EPSRC EP/E007748 and the EU CONNECT consortium www.brain-connect.eu.

\section{References}

1. Basser, P., Mattiello, J., LeBihan, D.: MR diffusion tensor spectroscopy and imaging. Biophys. J. 66 (1994)

2. Assaf, Y., Blumenfeld-Katzir, T., Yovel, Y., Basser, P.: AxCaliber: A Method for Measuring Axon Diameter Distribution from Diffusion MRI. MRM 59 (2008)

3. Barazany, D., Assaf, Y., Basser, P.: In vivo measurement of axon diameter distribution in the corpus callosum of rat brain. Brain 132 (2009)

4. Alexander, D.C., Hubbard, P.L., Hall, M.G., Moore, E.A., Ptito, M., Parker, G.J.M., Dyrby, T.B.: Orientationally invariant indices of axon diameter and density from diffusion MRI. NeuroImage (in press, 2010)

5. Alexander, D.C.: A general framework for experiment design in diffusion MRI and its application in measuring direct tissue-microstructure features. MRM 60 (2008)

6. O'Brien, T.E., Funk, G.M.: A gentle introduction to optimal design for regression models. Am. Stat. 57 (2003)

7. Zelinka, I., Lampinen, J.: SOMA - self-organizing migrating algorithm. In: 6th International Conference on Soft Computing (2002)

8. Gelderen, P.V., Despres, D., Zijl, P.C.M.V., Moonen, C.T.W.: Evaluation of restricted diffusion in cylinders phosphocreatine in rabbit leg muscle. JMR 103 (1994)

9. Szafer, A., Zhong, J., Gore, J.: Theoretical model for water diffusion in tissues. MRM 33 (1995)

10. Hall, M.G., Alexander, D.C.: Convergence and parameter choice for Monte-Carlo simulations of diffusion MRI. IEEE TMI 28 (2009)

11. Aboitiz, F., Scheibel, A., Fisher, R., Zaidel, E.: Fiber composition of the human corpus callosum. Brain Research 598 (1992) 